Draft Version November 2, 2018

Preprint typeset using $\mathrm{LATE}_{\mathrm{E}} \mathrm{X}$ style emulateapj v. 08/22/09

\title{
DISCOVERY OF BURST OSCILLATIONS IN THE INTERMITTENT ACCRETION-POWERED MILLISECOND PULSAR HETE J1900.1-2455
}

\author{
Anna L. Watts ${ }^{1}$, Diego Altamirano, Manuel linares, Alessandro Patruno, Piergiorgio Casella, Yuri \\ Cavecchi, Nathalie Degenaar, Nanda Rea, Paolo Soleri, Michiel van der Klis \& Rudy Wijnands \\ Astronomical Institute "Anton Pannekoek", University of Amsterdam, Kruislaan 403, 1098 SJ Amsterdam, the Netherlands \\ Draft version November 2, 2018
}

\begin{abstract}
We report the discovery of burst oscillations from the intermittent accretion-powered millisecond pulsar (AMP) HETE J1900.1-2455, with a frequency $1 \mathrm{~Hz}$ below the known spin frequency. The burst oscillation properties are far more similar to those of the non-AMPs and Aql X-1 (an intermittent AMP with a far lower duty cycle), than those of the AMPs SAX J1808.4-3658 and XTE J1814-338. We discuss the implications for models of the burst oscillation and intermittency mechanisms.

Subject headings: binaries: general, stars: individual (HETE J1900.1-2455), stars: neutron, stars: rotation, X-rays: bursts, X-rays: stars
\end{abstract}

\section{INTRODUCTION}

There are two ways in which we can measure the spin of rapidly rotating accreting neutron stars: via accretionpowered pulsations (due to magnetic channeling of infalling material), or burst oscillations (coherent pulsations seen during the thermonuclear explosions of accreted fuel that give rise to Type I X-ray bursts). The mechanism that generates the brightness asymmetries responsible for burst oscillations is not yet known. Once understood, however, it should shed light on the thermonuclear burning process and the composition of the outer layers of the neutron star (Strohmayer \& Bildsten 2006; Watts 2008).

Burst oscillations often drift upwards in frequency by a few $\mathrm{Hz}$ during a burst. However the stability of the asymptotic frequency for a particular source (Muno et al. 2002a), and the detection of the same frequencies in superbursts as well as Type I bursts (Strohmaver \& Markwardt 2002), suggested even at an early stage that burst oscillation frequency might be (to within a few $\mathrm{Hz}$ ) the spin frequency. This was confirmed in 2003 with the discovery of burst oscillations at or within a few $\mathrm{Hz}$ of the spin frequency in the accretion-powered millisecond pulsars (AMPs) SAX J1808.4-3658 and XTE J1814-338 (Chakrabarty et al. 2003; Strohmaver et al. 2003). The near identity of burst oscillation frequency and spin frequency is now the major constraint on models for the burst oscillation mechanism.

The AMPs are particularly useful when investigating the burst oscillation mechanism, since they are the only sources in which we can gauge the effects of the magnetic field and asymmetric fuel deposition. They are also the only stars where we can measure the size and sign of the small offset between the spin and burst oscillation frequency. However both SAX J1808.4-3658 and XTE J1814-338 have burst oscillations with quite atypical properties compared to the other sources (Section 3). It is not yet clear whether we are seeing a continuum of behavior (which could be explained by one burst oscillation mechanism) or completely separate classes.

${ }^{1}$ A.L.Watts@uva.nl
The picture has evolved in the last three years with the discovery of three intermittent AMPs: HETE J1900.1-2455, SAX J1748.9-2021 and Aql X-1 (Kaaret et al. 2006; Gallowav et al. 2007; Gavriil et al. 2007; Altamirano et al. 2008; Casella et al. 2008). Unlike the other AMPs these sources show accretionpowered pulsations only sporadically. What cause the intermittency is also not known, with models including sporadic obscuration of the magnetic poles (Göğüs et al. 2007 and references therein), wandering of the accretion funnel and hence the hot spot around the magnetic pole (Romanova et al. 2003, 2004; Lamb et al. 2008), or magnetic field burial (Cumming et al. 2001; Cumming 2008).

If the magnetic field is what makes the burst oscillations of the AMPs so different from those of the other stars, then an intermittency mechanism that affects the magnetic field may also influence burst oscillation properties. Burst oscillations from the intermittent AMPs (all of which burst) are therefore an important piece of the puzzle. Until now, however, only Aql X-1 has shown burst oscillations, at $\approx 0.5 \mathrm{~Hz}$ below the spin frequency (Zhang et al. 1998; Casella et al. 2008). The properties of the Aql X-1 burst oscillations are very similar to those of the non-AMPs: however this source has the lowest pulsation duty cycle of any of the intermittent AMPs. In this Letter we report the discovery of burst oscillations from the intermittent AMP HETE J1900.1-2455 (Watts et al. 2009), and consider the implications for the burst oscillation and intermittency mechanisms.

\section{BURST ANALYSIS}

HETE J1900.1-2455 was first detected in June 2005, and has remained in outburst ever since apart from a 3 week period of quiescence in 2007 (Degenaar et al. 2007). It was quickly identified as an AMP with a spin frequency of $377.3 \mathrm{~Hz}$, an orbital period of 83.3 minutes, and a low $\left(<0.1 M_{\odot}\right)$ mass companion (Kaaret et al. 2006). It is at a distance of $\approx 5 \mathrm{kpc}$, determined from Eddington limited X-ray bursts (Gallowav et al. 2008a). Accretionpowered pulsations of low fractional amplitude $(\lesssim 3 \%)$ were detected sporadically during the first two months of outburst, but have not been seen since (Galloway et al. 2007, 2008b). There is some evidence that the in- 
termittent appearance of the accretion-powered pulsations may be related to the occurrence of Type I X-ray bursts, but whether the relationship is causal is not clear (Galloway et al. 2007). From 2005-2008 several X-ray bursts from this source were detected by HETE-II, the Rossi X-ray Timing Explorer (RXTE) and SWIFT ${ }^{2}$. We searched the bursts detected by both the RXTE Proportional Counter Array (PCA) and the SWIFT Burst Alert Telescope (BAT) for oscillations, but found no significant signal.

\subsection{Burst oscillations}

During routine monitoring on April 22009 (08:57 UTC), RXTE detected another X-ray burst with both the PCA and the High Energy X-ray Timing Experiment (HEXTE). Timing analysis was conducted using $125 \mu \mathrm{s}$ time resolution $\mathrm{PCA}$ event mode data from the two active PCUs (PCUs 0 and 2). We used all photons in the 2-30 $\mathrm{keV}$ range, the band where burst emission exceeds the persistent level. The data were barycentered using the JPL DE405 and spacecraft ephemerides, with the source position of Fox (2005). Some event mode data overruns occurred in the burst peak, leading to short data gaps.

A dynamical power spectrum (Figure1) reveals strong burst oscillations during the initial decay of the burst, drifting upwards by about $1 \mathrm{~Hz}$. Selecting only data after $t=4 \mathrm{~s}$ in Figure 1 (the time of the final data gap), we find maximum Leahy power in the range 17-30 in 4 independent consecutive $2 \mathrm{~s}$ bins, an extremely robust detection. The oscillations fall below the detectability threshold when the frequency is $376.3 \mathrm{~Hz}, 1 \mathrm{~Hz}$ below the spin frequency. Although the binary ephemeris cannot be extended to 2009 (the errors are too large), orbital Doppler effects will shift the spin frequency by $0.009 \mathrm{~Hz}$ at most (Kaaret et al. 2006), so the offset in the frequencies is secure. A search for accretion-powered pulsations during the observation revealed no significant signals.

To study the pulse profile, we extracted data for the $8 \mathrm{~s}$ period ( $\mathrm{t}=4-12 \mathrm{~s}$ in Figure 1) during which the oscillations are detected. We fitted the frequency drift using a polynomial model to maximise the power. Power was maximised with a quadratic drift model, $\nu=\nu_{0}+\dot{\nu}_{0}\left(t-t_{0}\right)+\ddot{\nu}_{0}\left(t-t_{0}\right)^{2}$, which yielded a Leahy normalised power of 88 (the best linear model gave a maximum power of 78 , and higher order polynomial terms yielded no further improvement). Using the best fit quadratic frequency model we generated a folded profile. The persistent (non-burst) emission level was estimated from the $\sim 1000$ s prior to the burst. The resulting pulse profile is shown inset in Figure 11. The pulse is well fit with a simple sinusoid $\left(\chi^{2} /\right.$ dof $\left.=31 / 37\right)$, with no need for harmonic content. The fractional amplitude was $(3.5 \pm 0.3) \%$ Root Mean Square (RMS) (2-30 keV), comparable to the fractional amplitude of the accretion-powered pulsations of this source. The amplitude of the pulsations rises with energy, from $(3.1 \pm 0.5) \%$ RMS in the $2-5 \mathrm{keV}$ band to $(5.5 \pm 0.6) \%$ RMS in the 10-20 keV band. The burst oscillations also show hard lags, with the $10-20 \mathrm{keV}$ pulse lagging the $2-5$

\footnotetext{
2 Galloway et al. (2008b) list most of the bursts detected during 2005-2008. One additional burst during this period was recorded by RXTE on December 52007 (05:57 UTC), and there is a candidate burst in slew data on November 272007.
}

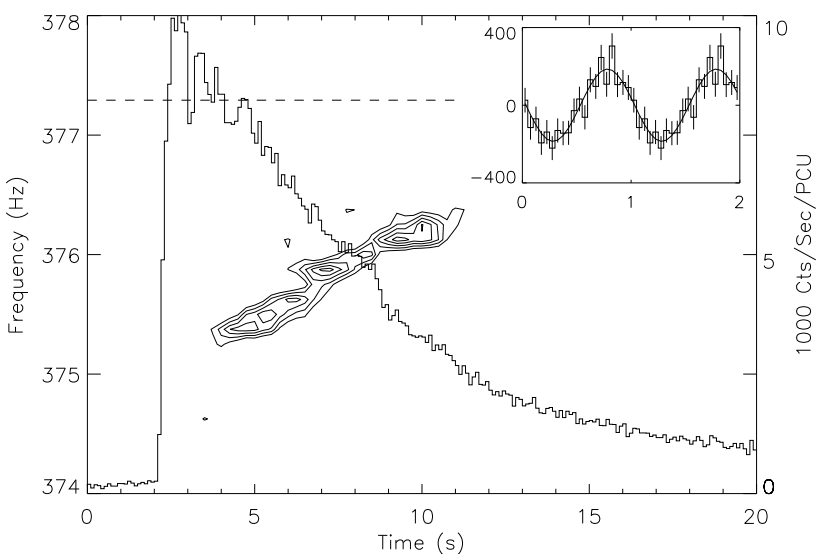

FIG. 1. - Lightcurve and dynamical power spectrum (2-30 keV) for the April 22009 burst. The dynamical power spectrum uses overlapping $4 \mathrm{~s}$ bins, with new bins starting at $0.25 \mathrm{~s}$ intervals. We use a Nyquist frequency of $2048 \mathrm{~Hz}$ and an interbin response function to reduce artificial drops in amplitude as the frequency drifts between Fourier bins (van der Klis 1989). The contours show Leahy normalized powers of 20-40, increasing in steps of 5 . The dashed line indicates the $377.3 \mathrm{~Hz}$ spin frequency. The inset shows the pulse profile of the burst oscillations for the $8 \mathrm{~s}$ interval where they are detected, folded with the best fit polynomial frequency model (counts per 0.05 cycle phase bin against phase in cycles), with the mean subtracted. The resulting profile is well fit by a simple sinusoid (overplotted) with no requirement for any harmonic content. Two cycles are shown for clarity.

keV pulse by $(0.09 \pm 0.03)$ cycles.

\subsection{Burst properties}

To study the characteristics of the burst containing the oscillations, we extracted spectra every $0.25 \mathrm{~s}$ from the PCA Event data (E_125us_64M_0_1s), which has 64 energy channels between 2 and $60 \mathrm{keV}$. We used data from PCUs 0 and 2, which were operating at the time of the $\mathrm{X}$-ray burst. We generated an instrument response matrix for each spectrum, and fitted it using XSPEC version 11.3.2. We added a $1 \%$ systematic error to the spectra and restricted the spectral fits to the energy range 2-25 $\mathrm{keV}$. We extracted spectra using 100s segments before and after the burst, and used them as background in our fits. We fitted each of the $0.25 \mathrm{~s}$ spectra with an absorbed (wabs, Morrison \& McCammon 1983) blackbody model (bbodyrad model in XSPEC). This approach, which takes account of the contribution of the persistent and background emission, is standard procedure in X-ray burst analysis (see for example Kuulkers et al. 2003).

The simple blackbody fit described above yields a peak luminosity of $3.6 \times 10^{38} \mathrm{ergs} \mathrm{s}^{-1}$, and a total energy of $2.7 \times 10^{39}$ ergs. The evolution of the temperature $T_{b b}$ and the radius $R_{b b}$ given by the spectral fits resembles those seen in photospheric radius expansion (PRE) bursts (Galloway et al. 2008a), i.e. (i) $R_{b b}$ reaches a local maximum close to the time of the peak flux, (ii) lower values of $R_{b b}$ were measured following the flux maximum and (iii) there is a local minimum in $T_{b b}$ at the same time as the maximum in $R_{b b}$. This conclusion (and the derived peak flux and fluence) should be treated with care, however, since the $\chi^{2} / d o f$ of the spectral fits can reach values as high as 4 or 5 . The problem stems from an excess at high energies. Similar issues were reported by 


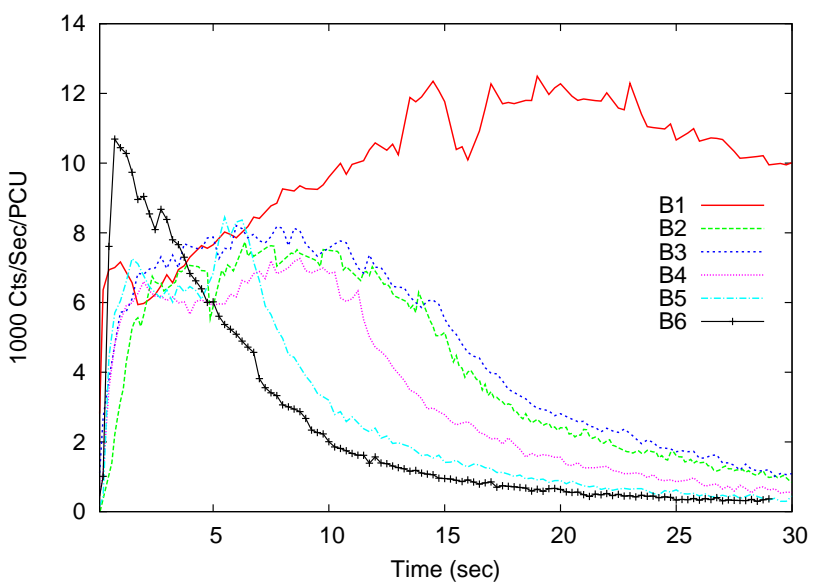

FIG. 2.- 2-25 keV lightcurves for the bursts recorded by the RXTE PCA from 2005-2009. Background and persistent emission have been subtracted and deadtime corrections applied (this was not done in Figure 1). The bursts are numbered according to their position in the color-color diagram (Figure 4). B6, the burst with the oscillations, has quite a different lightcurve.

Gallowav et al. (2008b) for other bursts from this source, and the physical cause is not yet understood. Adding a power law to our model, for example, reduces $\chi^{2} / d o f$ to below 2: the evolution of $T_{b b}$ and $R_{b b}$ in this case still suggests a PRE burst. Photospheric touchdown occurs $3.5-5 \mathrm{~s}$ after the start of the burst, consistent with the first detection of the burst oscillations.

The parameters of the April 2 burst do not differ dramatically from those of other bursts from HETE J1900, which also show evidence for PRE subject to uncertainties about the spectral fits (Galloway et al. 2008a,b). The burst with oscillations does not however have the extended or double peak structure exhibited by most of the other bursts (Figure 2). The burst lightcurve shows a fast rise, $\approx 0.4 \mathrm{~s}$ defined as in Galloway et al. (2008a). The decay can be modelled with a double exponential with decay timescales as of $7.3 \mathrm{~s}$ and $8.4 \mathrm{~s}$ respectively. Total burst duration is $\approx 60 \mathrm{~s}$ (burst end is defined as the time when flux falls to $1 \%$ of the peak flux, corrected for persistent emission).

\subsection{Persistent emission}

Figure 3 shows the intensity, defined as the countrate in the $2.0-16.0 \mathrm{keV}$ band, for all publicly available observations from 2005-2009. Figure 4 shows the corresponding color-color diagram. Data were deadtime corrected, background subtracted, and X-ray bursts removed. For each observation we calculated X-ray colors from the Standard2 data. We defined soft color as the ratio between count rates in the $3.5-6.0$ and $2.0-3.5 \mathrm{keV}$ bands and hard color as the ratio between count rates in the $9.7-16.0$ and $6.0-9.7 \mathrm{keV}$ bands. We normalized colors and intensity to the Crab values nearest in time (Kuulkers et al. 1994) and in the same PCA gain epoch (see for example van Straaten et al. 2003).

It is clear from Figures 3, 4 that the observation where the burst oscillations were detected was rather unusual. The burst happened when source intensity was at its highest recorded level $(\approx 63 \mathrm{mCrab})$ and when it was in the soft (banana) state. All previous bursts have been detected in harder states. Fitting the PCA spectrum with an absorbed disk-blackbody plus power-law model,

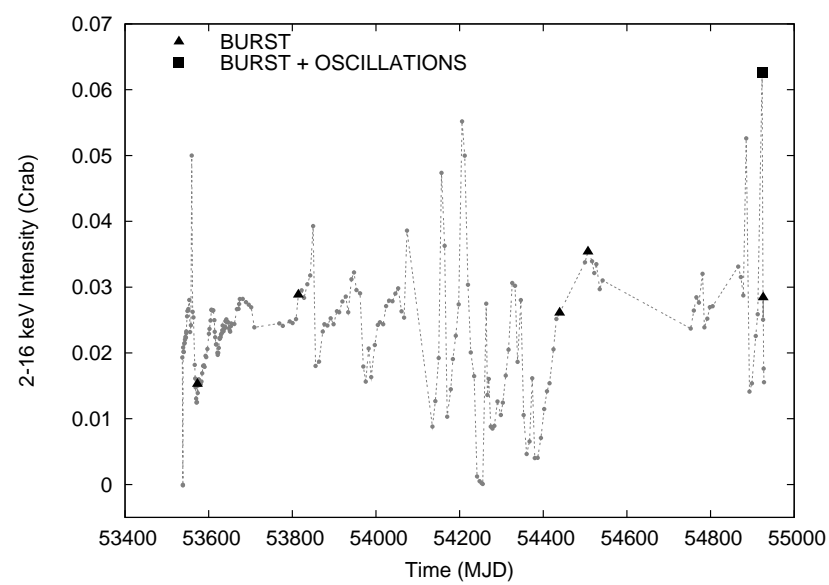

FIG. 3.- Average 2-16 keV intensity per RXTE observation for HETE J1900.1-2455 from 2005 to April 42009 (data from MarchOctober 2008 are not yet public). Observations containing bursts are marked in black: triangles (no oscillations), square (oscillations). We include the four confirmed bursts detected by the PCA from 2005-2008 (the candidate slew burst also occurs in the hard state, but is not shown), the burst with oscillations from April 2 2009 and an additional burst detected by the PCA on April 42009.

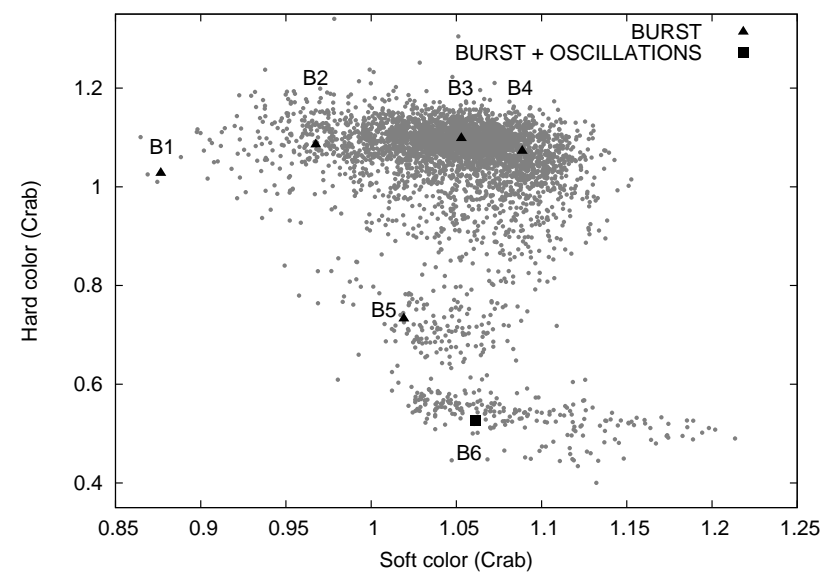

FIG. 4.- Color-color diagram for HETE J1900.1-2455 using all publicly available RXTE data from 2005 to April 42009 (excluding observations with intensities below $2 \mathrm{mCrab}$, see Figure 3). The source behaves like a typical atoll source (Hasinger \& van der Klis 1989). The grey dots show the colors computed from 160s segments of data. Observations containing bursts are marked in black: triangles (no oscillations), square (oscillations). Lightcurves for the bursts are shown in Figure 2

and assuming a standard bolometric correction factor of 2 (in't Zand et al. 2007) we find an unabsorbed bolometric flux of $3.5 \times 10^{-9} \mathrm{ergs} \mathrm{s}^{-1} \mathrm{~cm}^{-2}$ (interstellar absorption was fixed to $1.6 \times 10^{21} \mathrm{~cm}^{-2}$ ). At a distance of 5 $\mathrm{kpc}$, this corresponds to $4 \%$ of the Eddington luminosity if we assume $L_{\mathrm{Edd}}=2.5 \times 10^{38} \mathrm{erg} \mathrm{s}^{-1}$.

\section{DISCUSSION}

What causes burst oscillations is still not understood. Flame spread from a point should lead to asymmetries in the early phase of the burst. However, while this may explain the presence of burst oscillations during the rise, it is not sufficient to explain the continuing presence of large-scale asymmetries in the burst tail once the entire stellar surface has ignited (Strohmayer \& Bildsten 2006). This has led to the consideration of alternative 
models. Non-radial global oscillations in the surface layers of the star, excited by the flame spread, remain a promising possibility (Hevl 2004). In this case the brightness asymmetry would be caused by variations in ocean height associated with the mode. Inertial frame pattern speed can be very close to the spin rate, and cooling of the layers in the aftermath of the burst would naturally lead to frequency drift. The major problem of this model is that it overpredicts the size of the frequency drift compared to observations (Piro \& Bildsten 2005; Berkhout \& Levin 2008). Alternative possibilities like photospheric modes (Heyl 2004) or shear oscillations (Cumming 2005) also have shortcomings in their present form (Berkhout \& Levin 2008). Current efforts to resolve these problems are focusing on the role of the magnetic field, which can dominate the dynamics in the surface layers and is expected to have a large effect on surface modes.

If the magnetic field is the most important factor determining the properties of burst oscillations then this might lead to a natural explanation for the differences in the properties of the burst oscillations of SAX J1808.4-3658 and XTE J1814-338 (hereafter collectively referred to as SX: Chakrabarty et al. 2003; Strohmaver et al. 2003; Watts et al. 2005; Watts \& Strohmaver 2006) compared to those of the non-AMPs (Muno et al. 2002a, b, 2003, 2004; Galloway et al. 2008a). These differences can be summarized as follows. (1) SX show oscillations in all of their bursts, even in the hard state; the non-AMPs do not (oscillations are detected primarily although not exclusively in the soft state). (2) SX show oscillations throughout their bursts (except during PRE); the nonAMPs, in most cases, do not. (3) SX burst oscillations have amplitudes that fall with energy; the non-AMP oscillations have amplitudes that rise. (4) SX burst oscillations have detectable harmonic content; the non-AMPs have none. (5) Frequency drift in SX is either very fast or non-existent; in the non-AMPs it is much slower.

The magnetic field also plays an important role in models for intermittency of accretion-powered pulsations. In the obscuration and accretion stream wander models, the field is always present at the level necessary to channel the flow: but the accretion hot spot either wanders out of the line of sight or is obscured by magnetospheric ma- terial. In the magnetic burial model the field strength and geometry change as the field is suppressed by accretion. If magnetic field affects both intermittency and burst oscillations, then studying the latter may enable us to pinpoint the cause of the former.

In Aql X-1 the burst oscillations are similar in properties to those of the non-AMPs: however this source has an exceptionally low duty cycle so its intermittent pulsation episode may have been triggered by an extremely rare event. In HETE J1900.1-2455 the accretion-powered pulsations lasted for much longer. Its burst oscillations, however, also behave like those of the non-AMPs. This has important implications. If hot spot wander or obscuration models for intermittency are correct, then a field strong enough to channel infalling material cannot be the only factor causing the atypical burst oscillations of SAX J1808.4-3658 and XTE J1814-338. Some other factor, such as the presence of a strong temperature gradient around the magnetic pole, must also play a role in the burst oscillation mechanism (Watts et al. 2008). If on the other hand the field has been buried in HETE J1900.1-2455, then it must be screened to a depth where it is unable to affect the burst oscillation mechanism on the timescale of the burst. Detailed calculations will be required to resolve this issue, but if screening at the burning depth is not possible, then this could point to photospheric rather than oceanic modes as a cause of burst oscillations.

One other point of note is that in both Aql X-1 and HETE J1900.1-2455, burst oscillation frequency remains below spin frequency. Given the similarities in properties it seems probable that this is the case for non-AMPs as well. However it is not the case for SAX J1808.4-3658 and XTE J1814-338. In SAX J1808.4-3658 the burst oscillations are first detected below the spin frequency but rapidly overshoot it, settling $\sim 0.1 \mathrm{~Hz}$ above the spin frequency in the burst tail (Chakrabarty et al. 2003). In XTE J1814-338 the two frequencies are identical, and in fact burst oscillations and accretion-powered pulsations are coherent and phase-locked (Strohmayer et al. 2003; Watts et al. 2005, 2008). Any unified model of burst oscillations must be able to explain this diversity in the relationship between burst oscillation frequency and spin.

\section{REFERENCES}

Altamirano D. et al., 2008, ApJ, 674, L45

Berkhout R.G., Levin Y., 2008, MNRAS, 385, 1029

Casella P. et al.,2008, ApJ, 674, L41

Chakrabarty D. et al., 2003, Nature, 424, 42

Cumming A. et al., 2001, ApJ, 557, 958

Cumming A., 2005, ApJ, 630, 441

Cumming A., 2008, in A decade of accreting millisecond pulsars,

eds Wijnands R. et al., AIP Conference Proceedings, 1068, 152

Degenaar N. et al., 2007, Astronomer's Telegram \#1098

Fox D.B., 2005, Astronomer's Telegram \#526

Galloway D.K. et al., 2007, ApJ, 654, L73

Galloway D.K. et al., 2008, ApJS, 179, 360

Galloway D.K. et al., 2008, in A decade of accreting millisecond pulsars, eds Wijnands R. et al., AIP Conference Proceedings, 1068,55

Gavriil F.P. et al., 2007, ApJ, 669, L29

Göğüss E. et al., 2007, ApJ, 659, 580

Hasinger G., van der Klis M., 1989, A\&A, 225, 79

Heyl J., 2004, ApJ, 600, 939

in't Zand J.J.M. et al., 2007, A\&A, 465, 953

Kaaret P. et al., 2006, ApJ, 638, 963
Kuulkers E. et al., 1994, A\&A, 289, 795 Kuulkers E. et al., 2003, A\&A, 399, 663

Lamb F.K. et al., 2008, arXiv:0809.4016

Morrison R., McCammon D., 1983, ApJ, 270, 119

Muno M.P. et al., 2002, ApJ, 580, 1048

Muno M.P. et al., 2002, ApJ, 581, 550

Muno M.P. et al., 2003, ApJ, 595, 1066

Muno M.P. et al., 2004, ApJ, 608, 930

Piro A.L., Bildsten L., 2005, ApJ, 629, 438

Romanova M.M. et al., 2003, ApJ, 595, 1009

Romanova M.M. et al., 2004, ApJ, 610, 920

Strohmayer T.E., Markwardt C.B., 2002, ApJ, 577, 337

Strohmayer T.E. et al., 2003, ApJ, 596, L67

Strohmayer T., Bildsten L., 2006, in Compact stellar X-ray sources, eds Lewin W., van der Klis M., Cambridge Astrophysics Series 39, Cambridge, UK, p.113

van der Klis M., 1989, in Timing Neutron Stars, eds Ögelman H.

\& van den Heuvel E.J.P., Kluwer Academic/Plenum

Publishers, New York, USA, p.27

van Straaten S. et al., 2003, ApJ, 596, 1155

Watts A.L. et al., 2005, ApJ, 634, 547 
Watts A.L., Strohmayer T.E., 2006, MNRAS, 373, 769

Watts A.L., 2008, in A decade of accreting millisecond pulsars,

eds Wijnands R. et al., AIP Conference Proceedings, 1068, 199

Watts A.L. et al., 2008, ApJ, 688, L37
Watts A.L. et al., 2009, Astronomers' Telegram \#2004

Zhang W. et al., 1998, ApJ, 495, L9 